\title{
A Study on the Effectiveness of Machine Learning Algorithms in Early Prediction of Diabetics among Patients
}

\author{
R.K. Kavitha ${ }^{1 *}$ and W. Jai Singh ${ }^{2}$ \\ ${ }^{1,2}$ Department of Computer Applications, Kumaraguru College of Technology, Coimbatore, India
}

\begin{abstract}
Nowadays healthcare industry seems to generate enormous data which when analysed using appropriate machine learning algorithms and tools shall provide greater insights of it. Such analysis helps to discover unusual and difficult to diagnose diseases at an early stage thus promising an increased success rate of curing such diseases and reduced medical expenses. This investigation aims to construct a model which will be able to foresee the chances of occurrence of diabetes among patients with greatest precision. Among the various machine learning algorithms which can be used for classifying data, three were chosen for this study and are listed as follows: Decision Tree, Naïve Bayes and Multilayer Perceptron. All the three techniques were applied on the diabetic data set and their performances were analysed using various metrics. Also, the results were compared by varying the k-fold values. Early stage diabetes risk prediction dataset obtained from UCI machine learning repository was utilized in this research work. This study has positively displayed the ability of the predicting patients with early diabetic risks in a large dataset. Among the three classification techniques, multilayer perceptron seems to classify the patient as diabetic or not with a higher degree of accuracy and with a chosen $\mathrm{k}$-fold value of five and eight.
\end{abstract}

KEY WORDS: CLASSIFICATION, EARLY DIABETICS, MACHINE LEARNING, MEDICAL DIAGNOSIS, PREDICTION.

\section{INTRODUCTION}

Throughout the world, many people are being affected by Diabetes at an early age (Mohemaiti et al., 2017). Diabetes is considered as a chronic disease that affects the insulin production in humans thus affecting the entire body metabolism (Choubey et al., 2017). A spike in the blood glucose levels is observed because of this disease (Georga et al., 2013). Diabetes can damage vital parts of the human body. Increase in thirst and hunger, repeated urination are a few symptoms caused due to high blood glucose

\section{ARTICLE INFORMATION}

*Corresponding Author: kavitha.rk.mca@kct.ac.in Received 12th Oct 2020 Accepted after revision 9th Dec 2020 Print ISSN: 0974-6455 Online ISSN: 2321-4007 CODEN: BBRCBA

Thomson Reuters ISI Web of Science Clarivate Analytics USA and Crossref Indexed Journal

\section{Clarivate
Analytics}

NAAS Journal Score 2020 (4.31) SJIF: 2020 (7.728)

A Society of Science and Nature Publication,

Bhopal India 2020. All rights reserved.

Online Contents Available at: http//www.bbrc.in/

Doi: http://dx.doi.org/10.21786/bbrc/13.11/22 levels which may lead to complications if not treated at an early stage. Factors such as weight, height, insulin levels and genetic factors may cause diabetes in humans. Apart from the listed factors, the glucose concentration in the blood is considered as a major reason for this disease. The quick detection of the symptoms remains the only solution to reduce the risk of stroke, blood pressure, kidney diseases, foot, eye, and skin complications. With proper treatment and suggested lifestyle changes, several people with diabetes will be able to avoid or postpone the onset of complications.

Machine learning in the field of healthcare is gaining popularity nowadays and used for clinical decision making (Pradhan et al., 2012). Several machine learning algorithms can be employed to examine medical images to discover abnormalities, classify data into various classes based on several features and predict diseases at a very early stage. Classification is a task that uses machine learning algorithms to designate a class label to the data set. Classification is one of the most essential

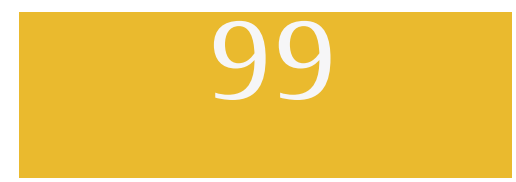


aspects of supervised learning. Several research studies have been conducted in various parts of the world to predict diseases using classification algorithms like SVM, Naive Bayes, J48, Decision Tree and so on (Kavakiotis et al., 2017; Vijayan 2015). This research focuses on predicting diabetics at an early stage by classifying the patients as diabetic or not based on the presence of several symptoms. Decision Tree, Naïve Bayes and Multilayer Perceptron algorithms were used in this study for early diabetic prediction. The performance of these algorithms was studied, and comparisons were drawn on various measures.

\section{MATERIAL AND METHODS}

Machine learning is a technique which when applied on medical datasets helps to detect and diagnose diseases in an effective manner (Dhomse Kanchan 2016). Diabetes, a universal disease which has created a serious impact on the society thus making machine learning as a priority in the medical research field. Several researchers have done a significant work in this field. Factors such as blood glucose levels, age, blood pressure, insulin, body mass index and skin thickness were used in a study to predict diabetes disease (Tarik et al., 2016). The contribution of adaboost and Bagging ensemble machine learning methods along with the support of decision tree technique to predict a patient as diabetic or non-diabetic was examined in a study (Perveen et al., 2016). The study has confirmed that adaboost technique performed better than the others. A ten-fold cross validation was performed on the data set, techniques like Logistic Regression, Naïve Bayes, and SVM was used in a study.

Figure 1: Workflow of Proposed model

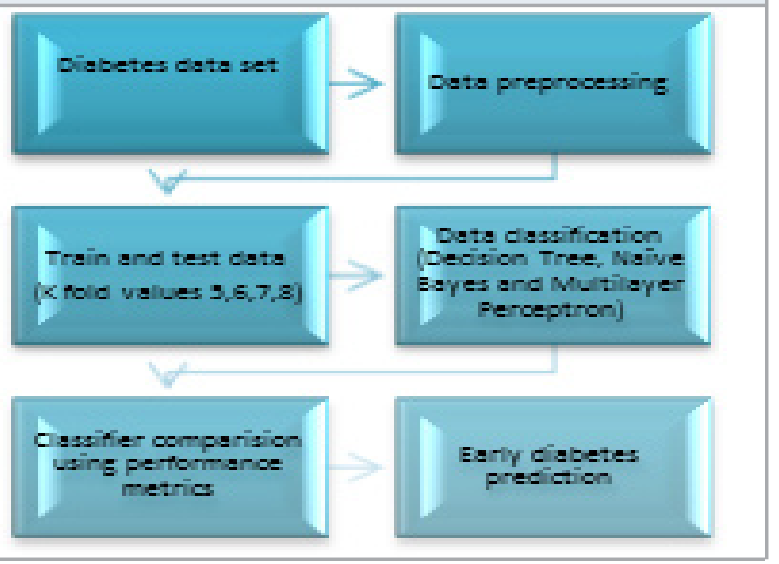

Table 1. Description of the Dataset

\begin{tabular}{|l|c|c|}
\hline Database Name & Total Attributes & Total Instances \\
\hline $\begin{array}{l}\text { Early stage diabetes } \\
\text { risk prediction dataset }\end{array}$ & 17 & 520 \\
\hline
\end{tabular}

It was concluded that SVM gave considerable accuracy with performance when compared to other methods
(Kavakiotis 2017). A model that used machine learning decision tree concept was devised for diabetes prediction with the aim of predicting diabetes among age group of people (Orabi et al., 2016). Acquired results were found to be satisfactory in forecasting diabetes at a certain age, with better accuracy applying Decision tree (Rokach 2008). Another researcher studied the performance of KNN and DISKR in reducing the storage space and concluded that outlier removal has increased both performance and accuracy of model (Song et al., 2017). Yet another study claimed that Naive Bayes provided better accuracy rate of 77.01\% in predicting diabetes (Rani 2016). Various popular machine learning classification methods such as Logistic Regression, Decision Tree, Naive Bayes as well as Artificial Neural Networks were used in classifying the diabetic risk among patients. To improve the strength of the proposed study, Bagging and Boosting techniques were employed. It was concluded that Random Forest algorithm displayed ideal results among the various other algorithms (Nongyao 2014).

Table 2. Attribute Description

\begin{tabular}{|l|c|}
\hline Attribute Description & Possible Values/range \\
\hline Age & Male, Female \\
\hline Sex & Yes, No \\
\hline Polyuria & Yes, No \\
\hline Polydipsia & Yes, No \\
\hline Sudden weight loss & Yes, No \\
\hline weakness & Yes, No \\
\hline Polyphagia & Yes, No \\
\hline Genital thrush & Yes, No \\
\hline Visual blurring & Yes, No \\
\hline Itching & Yes, No \\
\hline Irritability & Yes, No \\
\hline Delayed healing & Yes, No \\
\hline Partial paresis & Yes, No \\
\hline Muscle stiffness & Yes, No \\
\hline Alopecia & Yes, No \\
\hline Obesity & Positive, Negative \\
\hline Class & \\
\hline & \\
\hline
\end{tabular}

Figure 2: Overview of the dataset

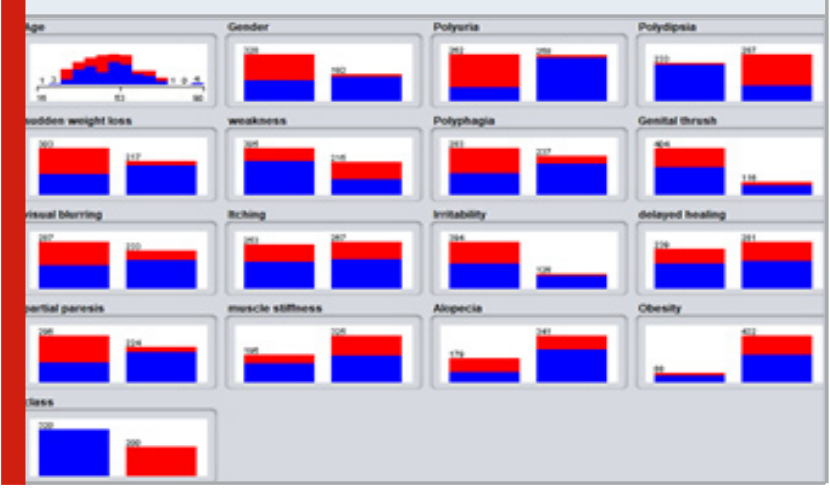


Proposed Work: The suggested research workflow is shown in figure 1 . The purpose of this study is to propose a classification model which can predict early stage diabetics among patients. The dataset was preprocessed to enhance the quality and to transform raw data into an understandable and readable format. Noise removal, handling of missing data was carried out. K-fold crossvalidation technique helped to split data as training and test sets. Data set was divided as $\mathrm{k}$ successive folds and every fold will be used once for validation whereas the left behind $\mathrm{k}-1$ folds is used as training set. K-fold values of 5,6,7 and 8 were used in the study.

Dataset: The suggested methodology is assessed on Early stage diabetes risk prediction dataset obtained from UCI machine learning repository. Dataset comprised of health details of 520 samples, including the sign and symptoms particulars of freshly diabetic or would be diabetic persons. The dataset contained 17 attributes whose description and possible values are shown in table 1 and 2. The number of patients classified as diabetic positive and diabetic negative for each attribute is shown in figure 2.

\section{Classification Techniques:}

Decision Tree: Decision Tree is considered as a supervised machine learning technique employed to classify data. The major intention of utilizing the technique here is to predict target class with the help of decision rules brought from earlier data (Agarwal et al., 2016). Internodes and nodes are used to predict and classify data. The root nodes are used to classify the various features found in the data. There may be branches appearing from the root node and the leaf nodes appearing from it classifies data. Information gain will be calculated for the attributes at each stage and the highest value of it will be used by the decision tree to choose each node (Rokach 2008).

\begin{tabular}{|c|c|c|}
\hline Performance Metric used & Meaning & Calculation \\
\hline Accuracy & $\begin{array}{l}\text { Lends overall classifier effectiveness, reveals } \\
\text { how many times the classifier } \\
\text { provided correct result in the given sample. }\end{array}$ & $\begin{array}{c}\mathrm{A}=(\mathrm{TP}+\mathrm{TN}) / \\
\text { (Total no of samples) }\end{array}$ \\
\hline Precision & $\begin{array}{l}\text { Ratio of true positives to the } \\
\text { total number of prediction as positives. }\end{array}$ & $\mathrm{P}=\mathrm{TP} /(\mathrm{TP}+\mathrm{FP})$ \\
\hline Recall & $\begin{array}{l}\text { Ratio of true positives to the } \\
\text { toral number of actual positives. }\end{array}$ & $\mathrm{R}=\mathrm{TP} /(\mathrm{TP}+\mathrm{FN})$ \\
\hline F-Measure & $\begin{array}{l}\text { Harmonic mean of precision and } \\
\text { recall, gives equal importance } \\
\text { to precision and recall. }\end{array}$ & $\mathrm{F}=2 /(1 /$ recall $+1 /$ precision $)$ \\
\hline ROC & $\begin{array}{l}\text { Graphic plot comparing classifier's } \\
\text { True Positive rates, False Positive rates. }\end{array}$ & Not Applicable \\
\hline
\end{tabular}

Table 4. Classifier: J48 - Decision Tree

\begin{tabular}{|l|c|c|c|c|c|c|}
\hline S. No & $\begin{array}{c}\text { No. of } \\
\text { K-fold }\end{array}$ & $\begin{array}{c}\text { True Positive } \\
\text { Rate }\end{array}$ & $\begin{array}{c}\text { False Positive } \\
\text { Rate }\end{array}$ & $\begin{array}{c}\text { Precision } \\
\text { value }\end{array}$ & $\begin{array}{l}\text { Recall } \\
\text { value }\end{array}$ & $\begin{array}{c}\text { ROC } \\
\text { Area }\end{array}$ \\
\hline 1 & 5 & 0.944 & 0.055 & 0.965 & 0.944 & 0.944 \\
\hline 2 & 6 & 0.952 & 0.043 & 0.953 & 0.952 & 0.962 \\
\hline 3 & 7 & 0.954 & 0.048 & 0.954 & 0.954 & 0.967 \\
\hline 4 & 8 & 0.948 & 0.053 & 0.948 & 0.948 & 0.962 \\
\hline
\end{tabular}

Table 5. Classifier: Naïve Bayes

\begin{tabular}{|l|c|c|c|c|c|c|}
\hline S. No & $\begin{array}{c}\text { No. of } \\
\text { K-fold }\end{array}$ & $\begin{array}{c}\text { True Positive } \\
\text { Rate }\end{array}$ & $\begin{array}{c}\text { False Positive } \\
\text { Rate }\end{array}$ & $\begin{array}{c}\text { Precision } \\
\text { value }\end{array}$ & $\begin{array}{c}\text { Recall } \\
\text { value }\end{array}$ & $\begin{array}{c}\text { ROC } \\
\text { Area }\end{array}$ \\
\hline 1 & 5 & 0.865 & 0.124 & 0.873 & 0.865 & 0.944 \\
\hline 2 & 6 & 0.875 & 0.116 & 0.881 & 0.875 & 0.947 \\
\hline 3 & 7 & 0.869 & 0.119 & 0.877 & 0.869 & 0.946 \\
\hline 4 & 8 & 0.877 & 0.114 & 0.883 & 0.877 & 0.947 \\
\hline
\end{tabular}


Table 6. Classifier: Multilayer Perceptron

\begin{tabular}{|l|c|c|c|c|c|}
\hline S. No & $\begin{array}{c}\text { No. of } \\
\text { K-fold }\end{array}$ & $\begin{array}{c}\text { True Positive } \\
\text { Rate }\end{array}$ & $\begin{array}{c}\text { False Positive } \\
\text { Rate }\end{array}$ & $\begin{array}{c}\text { Precision } \\
\text { value }\end{array}$ & $\begin{array}{c}\text { ROC } \\
\text { Area }\end{array}$ \\
\hline 1 & 5 & 0.960 & 0.036 & 0.961 & 0.960 \\
\hline 2 & 6 & 0.950 & 0.048 & 0.951 & 0.950 \\
\hline 3 & 7 & 0.962 & 0.039 & 0.962 & 0.962 \\
\hline 4 & 8 & 0.962 & 0.037 & 0.962 & 0.962 \\
\hline
\end{tabular}

Naïve Bayes: It can be understood that the entire features in Naive Bayes classification technique are not dependent and at the same time not related to each other. Here, the importance of a feature observed in a class will not affect other features. Being built on conditional probability, this technique is believed to be powerful in classifying data. It operates well on imbalanced dataset and data with missing values. The popular Bayes Theorem was employed by this classification technique (Mani et al., 2012).

Multilayer Perceptron: A multilayer perceptron (MLP) normally combines along with further perceptrons, piled in numerous layers with the idea of solving complicated problems. In this technique, more than one linear layer can be present (Rana et al., 2018). In a three-layer network, initial layer named as input layer, final called as output layer and the middle one as hidden layer. Normally, data is supplied into the input layer and obtained as output from the last layer. The hidden layer count can be increased as required in order to make the model more complex according the mission. The multilayer perceptron thus offers a nonlinear mapping amongst the input vector and output vector. The steps to be followed by perceptron are (1) Take the inputs, multiply by their weights and computes their sum (2) Adds a bias factor, the number one multiplied by a weight and (3) Feeds the sum all the way through activation function.

Figure 3: Classifier performance for K-Fold 5

$$
\mathrm{K}-\text { Fold }=5
$$

2

0

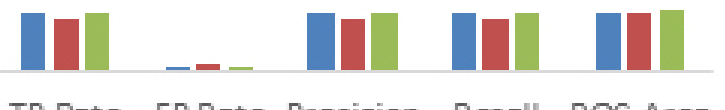

TP Rate FPRate Precision Recall ROC Area

Decision Tree

Näve Bayes

Multilayer Perceptron

Performance Metrics: To determine the implementation of classification technique, several metrics were used in the study. Accuracy, F-Measure, Recall, Precision and Receiver Operating Curve (ROC) measures were utilized classification algorithms used in the study namely Decision Tree, Naïve Bayes, and Multilayer Perceptron. Values of different metrics for the K-fold values of five,

Figure 4: Classifier performance for K-Fold 6

\section{$\mathrm{K}-\mathrm{Fold}=6$}

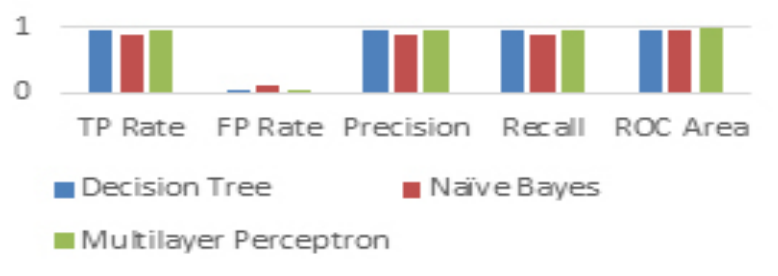

Figure 5: Classifier performance for K-Fold 7

\section{K-Fold $=7$}

2

0

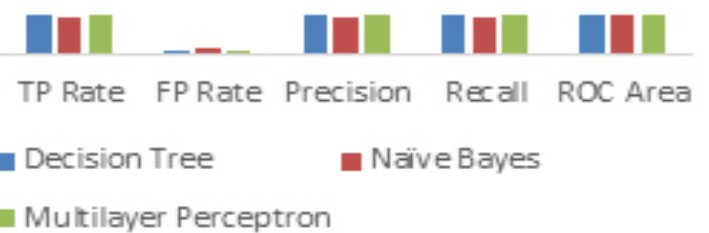

Figure 6: Classifier performance for K-Fold 8

\section{$\mathrm{K}-$ Fold $=8$}

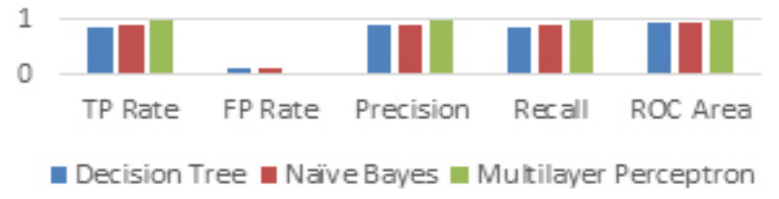

for data classification. The details of the accuracy measures are exhibited in table 3 .

\section{RESULTS AND DISCUSSION}

Tables 4, 5 and 6 represents the performance of three 
six, seven and eight are presented in the table. It can be interpreted from the results that the Multilayer Perceptron algorithm can predict the early diabetes with maximum precision, TP rate and ROC area when compared to other algorithms. Further, the performances of the three classifiers based on $\mathrm{k}$-fold values are plotted as a graph in figures $3,4,5$ and 6 .

Table 7. Comparison of classification techniques with error rates

\begin{tabular}{|l|l|c|c|c|c|c|c|c|}
\hline S. No & Model & $\begin{array}{c}\text { No. of K- } \\
\text { fold }\end{array}$ & $\begin{array}{c}\text { In-Correctly } \\
\text { Classified } \\
\text { Instances } \\
\text { Correctly } \\
\text { Classified }\end{array}$ & $\begin{array}{c}\text { Kappa } \\
\text { Statistic }\end{array}$ & $\begin{array}{c}\text { Mean } \\
\text { Absolute } \\
\text { error } \\
\text { value }\end{array}$ & $\begin{array}{c}\text { Root } \\
\text { mean } \\
\text { squared } \\
\text { error }\end{array}$ & $\begin{array}{c}\text { Relative } \\
\text { absolute } \\
\text { error (\%) }\end{array}$ \\
\hline 1 & J48 - Decision Tree & 5 & $491(94.4 \%)$ & $29(5.5 \%)$ & 0.883 & 0.077 & 0.232 & 16.28 \\
\hline 2 & J48 - Decision Tree & 6 & $495(95.1 \%)$ & $25(4.8 \%)$ & 0.899 & 0.063 & 0.214 & 13.4 \\
\hline 3 & J48 - Decision Tree & 7 & $496(95.38 \%)$ & $24(4.6 \%)$ & 0.902 & 0.059 & 0.208 & 12.65 \\
\hline 4 & J48 - Decision Tree & 8 & $493(94.8 \%)$ & $27(5.1 \%)$ & 0.890 & 0.067 & 0.223 & 14.31 \\
\hline 5 & Naïve Bayes & 5 & $450(86.5 \%)$ & $70(13.4 \%)$ & 0.722 & 0.150 & 0.320 & 31.8 \\
\hline 6 & Naïve Bayes & 6 & $45587.5 \%$ & $6512.5 \%$ & 0.742 & 0.147 & 0.316 & 31.0 \\
\hline 7 & Naïve Bayes & 7 & $45287 \%$ & $6813 \%$ & 0.730 & 0.149 & 0.319 & 31.6 \\
\hline 8 & Naïve Bayes & 8 & $45687.6 \%$ & $6412.3 \%$ & 0.745 & 0.147 & 0.317 & 31.0 \\
\hline 9 & Multilayer Perceptron & 5 & $49996 \%$ & $214 \%$ & 0.915 & 0.044 & 0.173 & 9.4 \\
\hline 10 & Multilayer Perceptron & 6 & $49495 \%$ & $265 \%$ & 0.895 & 0.053 & 0.193 & 11.21 \\
\hline 11 & Multilayer Perceptron & 7 & $50096 \%$ & $204 \%$ & 0.9191 & 0.045 & 0.179 & 9.65 \\
\hline 12 & Multilayer Perceptron & 8 & $50096 \%$ & $204 \%$ & 0.919 & 0.043 & 0.180 & 9.18 \\
\hline
\end{tabular}

A comparison of the classifiers with error rates are presented in the table 7. It may be inferred that Multilayer Perceptron algorithm performs well in correctly classifying the diabetic patients when compared to Decision Tree and naïve Bayes algorithms. The mean absolute error, root mean squared and relative absolute error rates for multilayer perceptron is comparatively low than the other algorithms used in the study. Also, it can be understood that the multilayer perceptron algorithm works better for chosen $\mathrm{k}$-fold values of 5 and 8 .

\section{CONCLUSION}

One of the crucial needs nowadays is the detection of diabetes at its initial phase. The proposed model helps to detect early diabetes among patients with high accuracy. The prediction outcomes of three machine learning classification algorithms were analysed considering various metrics and measures. Experiments were performed using early stage diabetes risk prediction dataset taken from UCI machine learning repository. The study findings suggest that Multilayer Perceptron algorithm outperforms the other two algorithms in classifying the data set as diabetic with more precision and less error rates. Also, this algorithm can be used to predict various other diseases in coming days.

Conflict of Interest: The authors declare no conflicts of interest.

\section{REFERENCES}

Agarwal V, Podchiyska T, Banda J.M et al (2016) Learning statistical models of phenotypes using noisy labeled training data Journal of the American Medical
Informatics Association Vol 23 No 6 Pages 1166-1173 Choubey D.K, Paul S, Kumar S (2017) Classification of Pima Indian diabetes dataset using naive bayes with genetic algorithm as an attribute selection Communication and Computing Systems Proceedings of the International Conference on Communication and Computing System (ICCCS 2016) Pages 451-455

Dhomse Kanchan B (2016) Study of Machine Learning Algorithms for Special Disease Prediction using Principal of Component Analysis 2016 International Conference on Global Trends in Signal Processing, Information Computing and Communication, IEEE Pages 5-10

Georga E.I, Protopappas V.C, Ardig`o D et al (2013) Multivariate Prediction of Subcutaneous Glucose Concentration in Type 1 Diabetes Patients Based on Support Vector Regression IEEE Journal of Biomedical Health Informatics Vol. 17 No 1 Pages 71-81

Kavakiotis I, Tsave 0, Salifoglou A et al (2017) Machine Learning and Data Mining Methods in Diabetes Research Computational and Structural Biotechnology Journal Vol 15, Pages 104-116

Mani S, Chen Y, Elasy T (2012) Type 2 Diabetes Risk Forecasting from EMR Data using Machine Learning AMIA Annual Symposium Proceedings Pages 606615

Mohemaiti P, Keyoumu Y et al (2017) Current situation and related risk factors of elderly type 2 diabetes mellitus with coronary heart disease in Hangzhou road community of the New Urban Area of Urumqi Chinese Journal of Gerontology Vol 37 No 21 Pages 
$5422-5424$

Nongyao N, Punnee S (2014) Ensemble Learning Model for Diabetes Classification Advanced Materials Research Vol 931-932 Pages 1427-1431

Orabi K.M, Kamal, Y.M, Rabah, T.M (2016) Early Predictive System for Diabetes Mellitus Disease Industrial Conference on Data Mining Springer Pages 420-427

Perveen S, Shahbaz M, Guergachi A et al (2016) Performance Analysis of Data Mining Classification Techniques to Predict Diabetes Procedia Computer Science Vol 82 Pages 115-12

Pradhan,P.M.A,Bamnote G.R.,Tribhuvan V et al (2012) A Genetic Programming Approach for Detection of Diabetes. International Journal of Computational Engineering Research Pages 91-94

Rana A, Singh Rawat A, Bijalwan et al (2018), "Application of Multi-Layer Perceptron Artificial Neural Network in the Diagnosis System: A Systematic Review International Conference on Research in Intelligent and
Computing in Engineering (RICE) San Salvador Pages $1-6$

Rani A, Swarupa, Jyothi S (2016) Performance analysis of classification algorithms under different datasets In Computing for Sustainable Global Development (INDIACom) Third International IEEE Conference Pages 1584-1589

Rokach L, Maimon Z.0 (2008) Data mining with decision trees: theory and applications Vol 13 World Scientific Publishing Co Toh Tuck Singapore Song, Yunsheng, Jiye Liang et al (2017) An efficient instance selection algorithm for $\mathrm{k}$ nearest neighbour regression Neurocomputing Pages 26-34

Tarik A, Rashid,S.M.A, Abdullah,R.M. (2016) An Intelligent Approach for Diabetes Classification Prediction and Description Advances in Intelligent Systems and Computing Pages 323-335

Vijayan,V.V, Anjali,C (2015) Prediction and diagnosis of diabetes mellitus A machine learning approach IEEE Recent Advances in Intelligent Computational Systems (RAICS) Pages 122-127 